\title{
BMJ Open Statin treatment and risk of recurrent venous thromboembolism: a nationwide cohort study
}

\author{
Cu Dinh Nguyen, ${ }^{1}$ Charlotte Andersson, ${ }^{1}$ Thomas Bo Jensen, ${ }^{1}$ Anne Gjesing, ${ }^{1}$ \\ Anne-Marie Schjerning Olsen, ${ }^{1}$ Carolina Malta Hansen, ${ }^{1}$ Harry Büller, ${ }^{2}$ \\ Christian Torp-Pedersen, ${ }^{3}$ Gunnar H Gislason ${ }^{4,5}$
}

To cite: Nguyen CD, Andersson C, Jensen TB, et al. Statin treatment and risk of recurrent venous thromboembolism: a nationwide cohort study. BMJ Open 2013;3:e003135. doi:10.1136/bmjopen-2013003135

- Prepublication history and additional material for this paper is available online. To view these files please visit the journal online (http://dx.doi.org/10.1136/ bmjopen-2013-003135)

Received 30 April 2013 Revised 2 October 2013 Accepted 9 October 2013

\section{(a) CrossMark}

${ }^{1}$ Department of Cardiology, Gentofte Hospital, University of Copenhagen, Denmark ${ }^{2}$ Department of Vascular Medicine, Academic Medical Center, Amsterdam, The Netherlands

${ }^{3}$ Department of Science and Technology, Institute of Health, Aalborg University, Denmark

${ }^{4}$ National Institute of Public Health, University of Southern, Denmark ${ }^{5}$ Faculty of Health and Medical Sciences, University of Copenhagen, Denmark

Correspondence to Dr Cu Dinh Nguyen; dinhcu@hotmail.com

\section{ABSTRACT}

Objectives: Statins may decrease the risk of primary venous thromboembolism (VTE), that is, deep vein thrombosis (DVT) and pulmonary embolism (PE) but the effect of statins in preventing recurrent VTE is less clear. The aim of this study was therefore to investigate the association between statin therapy and risk of recurrent VTE.

Design: A prospective cohort study.

Setting: All hospitals in Denmark.

Participants: All patients with a hospital diagnosis of VTE in Denmark during 1997-2009 associated with a warfarin or heparin prescription were identified.

Main outcome measures: Adjusted HR of recurrent hospitalised VTE (ie, fatal or non-fatal DVT or PE) associated with use of statins.

Results: 44330 patients with VTE were included in the study. Of these 3914 were receiving statin therapy at baseline. Patients receiving statins were older $(68 \pm 11$ compared to $62 \pm 18$ years), had more comorbidity and used more medications. The incidence rate for recurrent VTE was 24.4 (95\% Cl 22.8 to 26.2) per 1000 person-years among statin users and 48.5 (95\% Cl 47.4 to 49.7) per 1000 person-years among nonstatin users. Statin use was associated with a significantly lower risk of a recurrent VTE, adjusted HR $0.74(95 \% \mathrm{Cl} 0.68$ to 0.80$)$, compared with no statin use. The association between statin use and risk of recurrent VTE was significantly affected by age. Among younger individuals ( $\leq 80$ years), statin use was associated with lower risk of recurrent VTE, HR 0.70 $(95 \% \mathrm{Cl} 0.65$ to 0.76$)$ whereas in older individuals (>80 years) statin use was significantly associated with higher risk of recurrent VTE, HR $1.28(95 \% \mathrm{Cl} 1.02$ to 1.60), $p$ for interaction $=<0.0001$.

Conclusions: Statin use was associated with a decreased risk of recurrent VTE.

\section{INTRODUCTION}

Venous thromboembolism (VTE), that is, deep vein thrombosis (DVT) and pulmonary embolism (PE) are common diseases associated with high morbidity and mortality. Primary VTE has an incidence rate of

\section{Strengths and limitations of this study}

- We lacked data on chemotherapy, obesity, pregnancy and smoking.

- The main strength of our study was the large amount of individuals included.

- The main limitation was the observational nature of the study.

$1-2 / 1000$ person-years in Western populations $^{12}$ and more than $30 \%$ of patients with VTE experience at least one recurrent episode during the following 5-year period. ${ }^{3}{ }^{4}$ The risk of VTE is influenced by many temporary factors such as surgery, ${ }^{5}$ cancer and immobilisation, but if no such conditions are apparent, short-term secondary prophylaxis with anticoagulants may be insufficient in order to prevent recurrent VTE. A first episode of unprovoked VTE is nevertheless followed by 3-6 months of anticoagulant therapy with vitamin $\mathrm{K}$ antagonists in order to reduce the risk of recurrent episodes. The risk of major bleeding is the foremost complication of treatment and one of the main reasons for limiting treatment duration to 3-6 months. Statins have been shown to be associated with reduced risk of VTE and may therefore constitute a possible long-term supplement to antithrombotic treatment after VTE. ${ }^{7}$ The potential role of statins on the coagulation system is currently not clear, but statins may carry an antithrombotic effect by inhibiting the expression of tissue factor and thereby inhibit platelet activation. ${ }^{6}{ }^{8-12} \mathrm{~A}$ randomised controlled trial of rosuvastatin in the primary prevention of VTE, a meta-analysis, and several observational studies have shown a significant reduction in the risk of VTE associated with the use of statins ranging from $20 \%$ to $60 \% .^{13-20}$ However, another recent meta-analysis including unpublished trials showed no significant effect of statins on the primary risk of VTE. ${ }^{21}$ 
The effect of statin treatment on prevention of recurrent episodes in patients with a previous VTE is insufficiently investigated. Therefore, the aim of our study was to investigate if statin use could have a preventive effect on recurrent VTE in a nationwide cohort of patients with DVT or PE.

\section{METHODS}

\section{Data-sources}

In Denmark every resident is, at the time of birth or immigration, provided with a unique and permanent civil registration number, which enables linkage between nationwide administrative registers on the individual level. The Danish National Patient Register was established in 1978 and includes information on all hospitalisations in Denmark since 1978. At discharge, each admission is registered with one primary and if appropriate, one or more secondary diagnoses according to the International Classification of Diseases (ICD): the 10th revision (ICD-10). ${ }^{22} \quad 23$ The National Register of Medicinal Product Statistics ${ }^{24}$ holds data on every dispensed prescription from pharmacies in Denmark since 1995. Dispensed drugs are registered by the international Anatomical Therapeutic Classification (ATC) system along with information on date and amount dispensed. Information on vital status was obtained from the Danish Civil Registry ${ }^{25}$ and causes of death from the Danish Registry of Causes of Death. ${ }^{26}$

\section{Study population}

All patients with a hospital diagnosis of VTE between 1 January 1997 and 31 December 2009 (in patients and out patients) were identified. The VTE diagnoses comprised the following ICD-10 codes: I26 (pulmonary embolism), I80 (phlebitis and thrombophlebitis) and I82 (other venous embolism and thrombosis). Patients with any of I82.0 (Budd-Chiari syndrome), I80.0 (phlebitis and thrombophlebitis of superficial vessels of lower extremities) and I80.8 (phlebitis and thrombophlebitis of other sites) were excluded. To ensure a correct diagnosis of VTE we required that a diagnosis of VTE was followed by appropriate anticoagulation therapy and therefore excluded patients who did not claim a prescription of anticoagulation therapy from a pharmacy (heparin or warfarin/marcoumar) within 30 days of the diagnosis of VTE. The follow-up time started 90 days after the inpatient admission date (index date), which we assumed as the date of VTE, and patients were followed until the first occurrence of any of the following: hospitalisation with VTE diagnoses, emigration, death or 31 December 2009. The reason for starting follow-up 90 days after the hospital admission date was to avoid including any workup investigations related to the initial VTE event.

\section{Comorbidity}

Comorbidity was defined by hospitalisation diagnoses up to 1 year prior VTE diagnosis. The concomitant diagnoses (ICD-10 codes) of interest were ischaemic heart disease (I20, I23-I25), acute myocardial infarction (I21-I22), atrial flutter/fibrillation (I48), peripheral vascular disease (I70-I74), diabetes mellitus (E10-E14) and malignancies (C00-C97).

\section{Pharmacotherapy}

Use of the following drugs was identified: statins (ATC code C10AA), glucose-lowering medications (A10), nonsteroidal anti-inflammatory drugs (NSAIDs) (M01), hormone replacement therapy/oral contraceptives (G03), diuretics (C03), low-dose aspirin (B01AC06), clopidogrel (B01AC04), ACE inhibitor/angiotensin II antagonist (C09) and antipsychotics (N05A). Patients were defined as users at baseline if the prescription was redeemed 90 days before or 90 days after the index date. Patients were allowed to initiate and discontinue medications throughout follow-up and hence change exposure status during the study period.

The exposure status was updated approximately every 90 days. The daily dosage of medication is not recorded in the national prescription register. Treatment with vitamin $\mathrm{K}$ antagonists (B01AA) and statins were assessed for each day by considering the amount of claimed medications and claiming time interval for up to three consecutive prescriptions. On the basis of these assumptions, we calculated whether patients at any time had tablets available or not. Patients were considered exposed only while taking the medications. This method is described in more detail elsewhere. ${ }^{27}$ The minimal dose of warfarin is $1.25 \mathrm{mg}$ and maximal dose is $10 \mathrm{mg}$. Minimal dose of simvastatin, lovastatin, pravastatin, fluvastatin, atorvastatin and rosuvastatin are 5, 10, 10, 20, 10 and $5 \mathrm{mg}$, respectively. The maximal dose is $80 \mathrm{mg}$ for all subtypes of statins. The cut-off points for high dosage of simvastatin, lovastatin, pravastatin, fluvastatin and atorvastatin were $20 \mathrm{mg}$ and for rosuvastatin the cut-off point was $10 \mathrm{mg}$.

\section{Surgery}

From The Danish National Patient Register we obtained information on cardiovascular surgery, abdominal surgery and orthopaedic surgery to adjust for the risk of VTE associated with surgery in the multivariate regression analysis. Surgery was included as a time-varying covariate in the models and patients were considered exposed 30 days following the date of surgery.

\section{Study outcome}

A recurrent VTE was defined as a new inpatient or outpatient admission with the diagnosis of VTE (ICD-10 codes I26, I80 or I82, except I82.0, I80.0 and I80.8, which were excluded) together with a claimed prescription of anticoagulants (heparin or vitamin K antagonists) within 30 days before or after the diagnosis, or VTE coded as the primary or contributing cause of death in the death certificate (same ICD-codes as above). For the composite endpoint, VTE and individual 
endpoints, patients with DVT or PE, were followed until the first recurrent event of the type of interest, emigration, death or end of study (31 December 2009).

\section{Statistical analysis}

Discrete variables were compared using $\chi^{2}$ test and continuous variables were compared using the $\mathrm{t}$ test. We also calculated crude incidence rates for recurrent DVT, PE and VTE as numbers of events/1000 person-years. Multivariable Cox proportional hazard regression models were used to estimate HR for the recurrent DVT, PE and VTE associated with individual risk factors. All models included age, sex, ischaemic heart disease, acute myocardial infarction, atrial flutter/fibrillation, peripheral vascular disease, diabetes mellitus, malignancies, statins, warfarin, low-dose aspirin, clopidogrel, hormone replacement therapy/oral contraceptives, glucoselowering medications, diuretics, NSAIDs, ACEi/ARB, antipsychotic medications and surgery. In all models, medications and surgery were time-dependent variables, meaning that patients were only considered exposed when they were taking the drugs or 30 days following the date of surgery. Model assumptions-the linearity of continuous variables, the proportional hazards assumption and lack of interactions-were tested and found valid unless otherwise indicated. Tests for interactions (between statin and the different variables) were performed by inclusion of an interaction term in the analysis.

All calculations were performed using SAS V.9.2 (SAS Institute, Cary, North Carolina, USA). A p value $<0.05$ was regarded statistically significant. Adjusted numbers needed to treat were calculated by using the methods described by Bender et al. ${ }^{28}$

\section{RESULTS}

A total of 44330 patients with VTE were included in the study. Of these $3914(9.7 \%)$ received statin therapy and 40416 did not receive statin therapy at study baseline. The mean follow-up time was 1438 days for the entire study population. In total 225 patients emigrated during follow-up. The baseline characteristics of statin users and non-statin users are presented in table 1. More patients initiated statin therapy during follow-up and altogether 9869 patients received statin therapy in our study at some point in time. At baseline the majority $(81 \%)$ of statin users were treated with simvastatin (table 2). Patients with statin therapy were older $(68 \pm 11$ compared to $62 \pm 18$ years), had more concomitant diseases such as diabetes mellitus $(15 \%$ vs $4 \%)$ and peripheral artery disease $(7 \%$ vs $2 \%)$ and used more medications, particularly low-dose aspirin ( $50 \%$ vs $13 \%$ ), ACEi/ARB (50\% vs $15 \%)$ and glucose-lowering drug (20\% vs $5 \%)$, compared to patients without statin therapy.

Table 1 Baseline characteristic

\begin{tabular}{|c|c|c|}
\hline Characteristic & No statin therapy $(\mathrm{N}=40 \mathrm{416})$ & Statin therapy $(\mathrm{N}=3914)$ \\
\hline \multicolumn{3}{|l|}{ Age (years)-n (\%) } \\
\hline$\leq 50$ & $10278(25)$ & $264(7)$ \\
\hline$\overline{51}-60$ & $6623(16)$ & $575(15)$ \\
\hline $61-70$ & $8187(20)$ & $1190(30)$ \\
\hline $71-80$ & $8636(21)$ & $1304(33)$ \\
\hline$>80$ & $6692(17)$ & $581(15)$ \\
\hline Age, mean $( \pm S D)$, years & $62.1( \pm 17.7)$ & $68.4( \pm 11.3)$ \\
\hline Women-n (\%) & $20816(52)$ & $1810(46)$ \\
\hline \multicolumn{3}{|l|}{ Concomitant diseases* $-\mathrm{n}(\%)$} \\
\hline Ischaemic heart disease & $1639(4)$ & $868(22)$ \\
\hline Acute myocardial infarction & $343(1)$ & $249(6)$ \\
\hline Atrial flutter/fibrillation & $2196(5)$ & $375(10)$ \\
\hline Peripheral artery disease & $828(2)$ & $270(7)$ \\
\hline Diabetes mellitus & $1628(4)$ & $605(15)$ \\
\hline Malignancies & $3903(10)$ & $346(9)$ \\
\hline \multicolumn{3}{|l|}{ Concomitant medicationst-n (\%) } \\
\hline Low-dose aspirin & $5440(13)$ & $1954(50)$ \\
\hline Clopidogrel & $163(0.4)$ & $276(7)$ \\
\hline Hormone replacement therapy & $5281(13)$ & $335(9)$ \\
\hline ACEi/ARB & $6037(15)$ & $1939(50)$ \\
\hline Glucose-lowering medications & $1905(5)$ & $800(20)$ \\
\hline Diuretics & $13362(33)$ & $2140(55)$ \\
\hline Antipsychotic medication & $2376(6)$ & $190(5)$ \\
\hline Nonsteroidal anti-inflammatory drugs & $10223(25)$ & $989(25)$ \\
\hline
\end{tabular}


Table 2 Type of statin used at baseline

\begin{tabular}{lrc}
\hline & $\mathbf{n}$ & Per cent \\
\hline Any statin expose & 3911 & \\
Simvastatin & 3185 & 81.4 \\
Lovastatin & 54 & 1.4 \\
Pravastatin & 208 & 5.3 \\
Fluvastatin & 43 & 1.1 \\
Atorvastatin & 377 & 9.6 \\
Rosuvastatin & 81 & 2.1 \\
\hline
\end{tabular}

\section{Association between statins and risk of recurrent VTE}

The median follow-up time was 1078 days (IQR 3612271 days) for the entire population, 423 days (IQR 121-1127 days) for those with recurrent VTE and 1285 days (IQR 491-2494 days) for those without recurrent VTE. Recurrent VTE occurred in 8264 (18\%) patients, of which 5320 had a recurrent DVT and 3744 had a recurrent PE. The incidence rate of recurrent VTE, DVT and PE are presented in table 3.

For the VTE endpoint statin use was associated with a lower risk of a recurrent VTE, unadjusted HR 0.75 (95\% CI 0.70 to 0.81 ; table 4 ), adjusted for age and sex, HR 0.74 (95\% CI 0.68 to 0.79$)$ and adjusted for all variables, HR 0.74 (95\% CI 0.68 to 0.80). A significant interaction was seen between statins and age, $p \leq 0.0001$. Statins were associated with a substantially lower risk of recurrent VTE among younger patients, compared with older patients. A significant interaction was also seen between statins and gender, $\mathrm{p} \leq 0.0001$. Statin use was associated with lower HR in men, compared with women, HR 0.65 (95\% CI 0.59 to 0.72 ) vs HR 0.87 (95\% CI 0.78 to 0.98 ). Further, a significant interaction was also found between statin use and low-dose aspirin, $\mathrm{p}=0.03$. Statin use was associated with significantly lower HR of VTE if administered without low-dose aspirin than if administered with low-dose aspirin, HR 0.70 (95\% CI 0.63 to 0.76 ) compared to 0.82 (95\% CI 0.72 to 0.93 ).

Analysing each endpoint separately, we found that statin use was associated with significantly lower risk of a recurrent DVT, HR 0.66 (95\% CI 0.59 to 0.71 ), as well as recurrent PE, HR 0.87 (95\% CI 0.78 to 0.97 ), compared to no statin use (table 4 ). The adjusted numbers needed to treat among statin users were 79 for VTE, 392 for PE and 97 for DVT.

A dose-response analysis revealed no significant differential association between low-dose statin use, HR 0.76 (95\% CI 0.68 to 0.85 ) and high-dose statin use, HR 0.71 (95\% CI 0.64 to 0.79 ) on the risk of a recurrent VTE.

\section{DISCUSSION}

In our nationwide study comprising over 40000 patients with prior VTE, statin use was associated with a significant reduction in the occurrence of recurrent VTE. This was found for recurrent DVT as well as for recurrent PE. This is the first study to evaluate the VTE-prophylactic

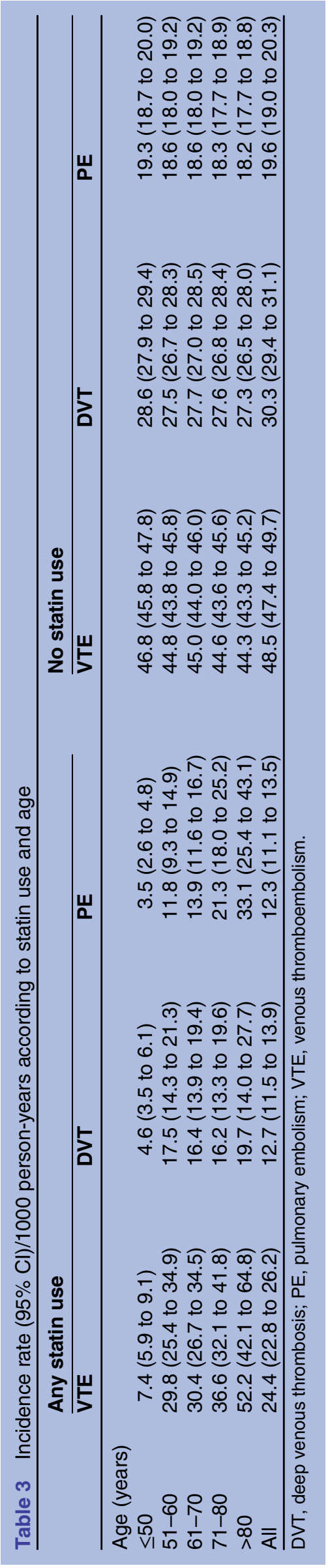


Table 4 HRs of venous thromboembolism associated with statin use

\begin{tabular}{|c|c|c|c|}
\hline Outcome & Unadjusted HR $(95 \% \mathrm{Cl})$ & Adjusted* HR (95\% Cl) & Adjusted† HR (95\% Cl) \\
\hline Venous thromboembolism & $0.75(0.70$ to 0.81$)$ & 0.74 (0.68 to 0.79$)$ & $0.74(0.68$ to 0.80$)$ \\
\hline Deep venous thrombosis & 0.61 (0.55 to 0.67$)$ & $0.63(0.57$ to 0.70$)$ & 0.66 (0.59 to 0.71$)$ \\
\hline Pulmonary embolism & $0.93(0.84$ to 1.04$)$ & 0.86 (0.77 to 0.95$)$ & 0.87 (0.78 to 0.97$)$ \\
\hline
\end{tabular}

effect of statin among participants with previous VTE on such a large population. Whether statins can be used as prophylaxis in patients with a previous VTE is an upcoming discussion. To our knowledge, two previous observational studies have focused on the influence of statins on the risk of recurrent thromboembolic events. Biere-Rafi et al recently used a Dutch population-based registry of pharmacy records linked with hospital discharge records and found that among 3039 patients with PE statin use was associated with decreased risk of recurrent pulmonary embolism (HR 0.50 (95\% CI 0.36 to 0.70$)) .{ }^{29}$ Delluc et al investigated the association between statin use and recurrent VTE on a small population of 432 participants and found no association. ${ }^{30}$

There are six subtypes of statins available on the market and it has not been clear if any reduction of VTE should be a class effect of statins or if it may differ between agents. In our study $80 \%$ of statin users were treated with simvastatin. We could therefore not explore a potential difference in effects between statins. However, a previous case-control study by Ramacharan et $a l,{ }^{19}$ with 4538 cases of first time VTE, verified by scanning and with the case's partners as controls, found that simvastatin, pravastatin and atorvastin were associated with lower risk of VTE. Doggens $e t a l^{16}$ found simvastatin to reduce the risk of VTE, but they were unable to find the same association for pravastatin. The recent study by Biere-Rafi et al showed that statins with the strongest potency, that is, atorvastatin and rosuvastatin, were associated with the greatest protection against a recurrent PE. In our study, however, we could not confirm such a differential association nor could we identify a doseresponse relation between statin dosages and the risk of VTE. The lack of dose-response relationship may be explained by the fact that the maximum effect of statins on the risk of VTE is reached already at low dose of statins. Further studies are warranted to investigate this subject.

We found an interesting modification of the effects associated with statins as dependent on age, and the antithrombotic effect of statins seemed to decrease with increasing age. Statin use among patients over 80 years of age was not significantly associated with a lower risk of recurrent VTE. Notably, increasing age in itself seemed to be protective against a recurrent VTE in our study. An explanation for the limited effect associated with statins in elderly patients could therefore be due to a lower baseline risk of VTE among elderly, compared with younger individuals. Another reason could be that patients over 80 have lower exposure to statins, by being less likely to be prescribed with statins, as seen in table 1 , or had lower compliance compared to those under 80. However, lower compliance is not likely to be a major reason, since a previous study has shown that elderly patients had better compliance with statins compared to younger patients. ${ }^{31}$

Interestingly, a recent randomised study, Aspirin for preventing the recurrence of venous thromboembolism (WARFASA) study, found that aspirin was effective in preventing unprovoked recurrent VTE after discontinuation of vitamin $\mathrm{K}$ antagonists. ${ }^{32}$ We investigated if adding statins to low-dose aspirin may be effective in reducing the risk further and we found that, although to a significantly less degree, statins were associated with lowered risks of VTE also among patients receiving aspirin. Further, our data suggest that statins may be as effective as aspirin in preventing VTE in patients with prior VTE and because they carry a lower risk of bleeding they may constitute an attractive alternative to aspirin in some patients. Ultimately, a randomised trial investigating the effect of aspirin, statin and the combination of the two agents for secondary prophylaxis after VTE should therefore be performed.

The exact mechanism underlying any protective effects of statins on risk of VTE is unknown. Some believe statins may carry an antithrombotic effect by inhibiting the expression of tissue factor and thereby inhibiting platelet activation. ${ }^{6}{ }^{8-12}$ Others have earlier suggested that the effect of statin beside the cholesterol lowering effect is due to 'healthy user effect'. 33 '34 Thus, persons who receive treatment with statins have a healthier lifestyle or/and a lower risk profile. In our population we cannot rule out a 'healthy user effect', although a recent Danish paper suggested that statin users do not have healthier lifestyle compare with non-statin users. ${ }^{35}$

\section{Strengths and limitations}

The main strength of our study was the large amount of individuals included. The Danish National Patient Registry, as well as the National Prescription Registry have proven to be accurate in other settings ${ }^{36} 37$ and the validity of the inpatient diagnoses of VTE has been investigated by Severinsen et al, who found a positive predictive value of $75 \%(95 \%$ CI $71.9 \%$ to $77.9 \%) .{ }^{38}$ The 
validity of outpatient diagnoses is unknown. To increase the likelihood of a correct VTE diagnosis we therefore only included patients with VTE diagnoses together with redemption of a prescription of anticoagulants (heparin or warfarin/marcoumar). Still we cannot exclude that the initial diagnosis of VTE was not in error because of the presence of other diseases (cellulitis, arthritis) mimicking VTE. Also statin users could have been taking dietary supplements or other over-the-counter medications not available in the records. These might have influenced the VTE recurrence rate.

The main limitation was the observational nature of the study. We tried to identify the most important risk factors for VTE and adjusted for them in our multivariable analyses. However, we lacked data on, for example, chemotherapy, obesity, pregnancy and recent trauma, which may have influenced our results. Unfortunately, we also lacked access to data on smoking. However, a meta-analysis from Circulation ${ }^{39}$ indicates that smoking does not increase the risk of VTE. But smokers are less likely to be exposed to statins. ${ }^{40}$ Smoking could have confounded our results and have led to overestimating the effect of statin use on the risk of VTE. Smoking is a well-known cardiovascular risk factor and by adjusting our analyses with cardiovascular diseases and cardiovascular medications we have tried to minimise the confounding effects of smoking on our results. Finally, low-dose aspirin and NSAIDs are available as over-the-counter medications; hence some patients may have been misclassified as non-users. The amount of over-the-counter low-dose aspirin in Denmark is probably low since low-dose aspirin, which is used almost exclusively for secondary prevention of cardiovascular disease, is generally prescribed by physicians, being reimbursable by up to $85 \%$ through the National Health Insurance Programme.

The observational nature of our study makes it difficult to draw a definite conclusion on whether or not statins lower the risk of recurrent VTE, but our results indicate that statins could prevent recurrent episodes of VTE.

\section{CONCLUSION}

In our study with over 40000 patients with a previous VTE, we found that the use of statins was associated with significantly lowered risk of a recurrent VTE compared to no use of statins. Further studies on the effect of statins on prevention of recurrent VTE episodes are warranted. In particular a randomised clinical trial investigating the effect of statins on the recurrence of VTE is needed to establish the effect of statins in this clinical context.

Contributors CDN was responsible for designing the study, collecting, analysing and interpreting the data and writing the manuscript. GHG and $\mathrm{CA}$ contributed by collecting, analysing and interpreting the data and critically revising the manuscript. TBJ, AG, AMSO, CMH, HB and CT-P contributed to the study by interpreting data and critically revising the manuscript. All approved the final manuscript. CDN is the guarantor.
Funding This research received no specific grant from any funding agency in the public, commercial or not-for-profit sectors.

\section{Competing interests None.}

Ethics approval Retrospective register studies do not require ethical approval in Denmark.

Provenance and peer review Not commissioned; externally peer reviewed.

Data sharing statement No additional data are available.

Open Access This is an Open Access article distributed in accordance with the Creative Commons Attribution Non Commercial (CC BY-NC 3.0) license, which permits others to distribute, remix, adapt, build upon this work noncommercially, and license their derivative works on different terms, provided the original work is properly cited and the use is non-commercial. See: http:// creativecommons.org/licenses/by-nc/3.0/

\section{REFERENCES}

1. Fowkes FJ, Price JF, Fowkes FG. Incidence of diagnosed deep vein thrombosis in the general population: systematic review. Eur $J$ Vasc Endovasc Surg 2003;25:1-5.

2. Heit JA. The epidemiology of venous thromboembolism in the community: implications for prevention and management. $J$ Thromb Thrombolysis 2006;21:23-9.

3. Nuttall M, van der Meulen J, Emberton M. Charlson scores based on ICD-10 administrative data were valid in assessing comorbidity in patients undergoing urological cancer surgery. J Clin Epidemiol 2006;59:265-73.

4. Christiansen SC, Cannegieter SC, Koster T, et al. Thrombophilia, clinical factors, and recurrent venous thrombotic events. JAMA 2005;293:2352-61.

5. Surgically confirmed gallbladder disease, venous thromboembolism, and breast tumors in relation to postmenopausal estrogen therapy. A report from the Boston Collaborative Drug Surveillance Program, Boston University Medical Center. New Eng J Med 1974;290:15-19.

6. Undas A, Brummel-Ziedins KE, Mann KG. Statins and blood coagulation. Arterioscler Thromb Vasc Biol 2005;25:287-94.

7. Kaba NK, Francis CW, Moss AJ, et al. Effects of lipids and lipid-lowering therapy on hemostatic factors in patients with myocardial infarction. J Thromb Haemost 2004;2:718-25.

8. Mason RP, Walter MF, Day CA, et al. Intermolecular differences of 3-hydroxy-3-methylglutaryl coenzyme a reductase inhibitors contribute to distinct pharmacologic and pleiotropic actions. Am J Cardiol 2005;96:11F-23F.

9. Dietzen DJ, Page KL, Tetzloff TA, et al. Inhibition of 3-hydroxy-3methylglutaryl coenzyme A (HMG CoA) reductase blunts factor VIIa/ tissue factor and prothrombinase activities via effects on membrane phosphatidylserine. Arterioscler Thromb Vasc Biol 2007;27:690-6.

10. Undas A, Brummel KE, Musial J, et al. Simvastatin depresses blood clotting by inhibiting activation of prothrombin, factor V, and factor XIII and by enhancing factor Va inactivation. Circulation 2001;103:2248-53

11. Undas A, Celinska-Lowenhoff M, Brummel-Ziedins KE, et al. Simvastatin given for 3 days can inhibit thrombin generation and activation of factor $\mathrm{V}$ and enhance factor $\mathrm{Va}$ inactivation in hypercholesterolemic patients. Arterioscler Thromb Vasc Biol 2005;25:1524-5.

12. Violi F, Calvieri C, Ferro D, et al. Statins as antithrombotic drugs. Circulation 2013;127:251-7.

13. Glynn RJ, Danielson E, Fonseca FA, et al. A randomized trial of rosuvastatin in the prevention of venous thromboembolism. New Engl J Med 2009;360:1851-61.

14. Squizzato A, Galli M, Romualdi E, et al. Statins, fibrates, and venous thromboembolism: a meta-analysis. Eur Heart $J$ 2010;31:1248-56.

15. Ray JG, Mamdani M, Tsuyuki RT, et al. Use of statins and the subsequent development of deep vein thrombosis. Arch Int Med 2001;161:1405-10.

16. Doggen CJM, Lemaitre $\mathrm{RN}$, Smith $\mathrm{NL}$, et al. HMG CoA reductase inhibitors and the risk of venous thrombosis among postmenopausal women. J Thromb Haemost 2004;2:700-1.

17. Grady D, Wenger NK, Herrington D, et al. Postmenopausal hormone therapy increases risk for venous thromboembolic disease. The Heart and Estrogen/progestin Replacement Study. Ann Intern Med 2000;132:689-96.

18. Lacut K, Le Gal G, Abalain JH, et al. Differential associations between lipid-lowering drugs, statins and fibrates, and venous thromboembolism: role of drug induced homocysteinemia? Thromb Res 2008;122:314-19. 
19. Ramcharan AS, Van Stralen KJ, Snoep JD, et al. HMG-CoA reductase inhibitors, other lipid-lowering medication, antiplatelet therapy, and the risk of venous thrombosis. J Thromb Haemost 2009;7:514-20.

20. Sorensen HT, Horvath-Puho E, Sogaard KK, et al. Arterial cardiovascular events, statins, low-dose aspirin and subsequent risk of venous thromboembolism: a population-based case-control study. J Thromb Haemost 2009;7:521-8.

21. Rahimi K, Bhala N, Kamphuisen $\mathrm{P}$, et al. Effect of statins on venous thromboembolic events: a meta-analysis of published and unpublished evidence from randomised controlled trials. PLoS Med 2012;9:e1001310.

22. Lynge E, Sandegaard JL, Rebolj M. The Danish National Patient Register. Scand J Public Health 2011;39(7 Suppl):30-3.

23. Mason K, Thygesen LC, Stenager E, et al. Evaluating the use and limitations of the Danish National Patient Register in register-based research using an example of multiple sclerosis. Acta Neurol Scand 2012;125:213-17.

24. Kildemoes HW, Sorensen HT, Hallas J. The Danish National Prescription Registry. Scand J Public Health 2011;39(7 Suppl):38-41.

25. Pedersen CB. The Danish Civil Registration System. Scand J Public Health 2011;39(7 Suppl):22-5.

26. Helweg-Larsen K. The Danish Register of Causes of Death. Scand J Public Health 2011;39(7 Suppl):26-9.

27. Merie C, Kober L, Skov Olsen P, et al. Association of warfarin therapy duration after bioprosthetic aortic valve replacement with risk of mortality, thromboembolic complications, and bleeding. JAMA 2012;308:2118-25.

28. Bender R, Blettner M. Calculating the "number needed to be exposed" with adjustment for confounding variables in epidemiological studies. J Clin Epidemiol 2002;55:525-30.

29. Biere-Rafi S, Hutten BA, Squizzato A, et al. Statin treatment and the risk of recurrent pulmonary embolism. Eur Heart $J$ 2013;34:1800-6.

30. Delluc A, Tromeur C, Le Moigne E, et al. Lipid lowering drugs and the risk of recurrent venous thromboembolism. Thromb Res 2012;130:859-63.
31. Gislason GH, Rasmussen JN, Abildstrom SZ, et al. Long-term compliance with beta-blockers, angiotensin-converting enzyme inhibitors, and statins after acute myocardial infarction. Eur Heart $J$ 2006;27:1153-8.

32. Becattini C, Agnelli G, Schenone A, et al. Aspirin for preventing the recurrence of venous thromboembolism. New Engl J Med 2012;366:1959-67.

33. Brookhart MA, Patrick AR, Dormuth C, et al. Adherence to lipid-lowering therapy and the use of preventive health services: an investigation of the healthy user effect. $A m \mathrm{~J}$ Epidemiol 2007;166:348-54.

34. Glynn RJ, Schneeweiss S, Wang PS, et al. Selective prescribing led to overestimation of the benefits of lipid-lowering drugs. J Clin Epidemiol 2006;59:819-28.

35. Thomsen RW, Nielsen RB, Norgaard M, et al. Lifestyle profile among statin users. Epidemiology 2013;24:619-20.

36. Madsen M, Davidsen M, Rasmussen S, et al. The validity of the diagnosis of acute myocardial infarction in routine statistics: a comparison of mortality and hospital discharge data with the Danish MONICA registry. J Clin Epidemiol 2003;56:124-30.

37. Gaist D, Andersen M, Aarup AL, et al. Use of sumatriptan in Denmark in 1994-5: an epidemiological analysis of nationwide prescription data. Br J Clin Pharmacol 1997;43: 429-33.

38. Severinsen MT, Kristensen SR, Overvad K, et al. Venous thromboembolism discharge diagnoses in the Danish National Patient Registry should be used with caution. J Clin Epidemiol 2010;63:223-8.

39. Ageno W, Becattini C, Brighton T, et al. Cardiovascular risk factors and venous thromboembolism: a meta-analysis. Circulation 2008;117:93-102.

40. Rabinowich L, Steinvil A, Leshem-Rubinow E, et al. Adherence to statins is associated with reduced incidence of idiopathic venous thromboembolism: real-life data from a large healthcare maintenance organisation. Heart 2012;98: 1817-21. 